\title{
ESD Characterization of planar InGaAs devices
}

\author{
Z. Ji ${ }^{(1)}$, D. Linten, R. Boschke, G. Hellings, S. H. Chen, A. Alian, D. Zhou, Y. Mols, T. Ivanov, J. Franco, B. Kaczer, \\ X. Zhang ${ }^{(1)}$, R. Gao ${ }^{(1)}$, J. F. Zhang ${ }^{(1)}$, W. Zhang ${ }^{(1)}$, N. Collaert and G. Groeseneken \\ IMEC, Leuven B3001, Belgium, ${ }^{(1)}$ School of Engineering \\ Liverpool John Moores University, Liverpool L3 3AF, UK
}

\begin{abstract}
We present a comprehensive study of ESD reliability (TLP) on planar nMOSFETs with $\mathrm{In0}_{.53} \mathrm{Ga}_{0.47} \mathrm{As}$ as the channel material. Two types of traps are found during ESD stress. They are formed through independent mechanisms: transient Ef-lowering induced pre-existing e-traps discharging in the gate stack and hot hole induced e-traps generation through impact ionization in the InP buffer. These two types of traps explain the observed walk-out of off-state channel leakage current as well as the two-stage current conduction phenomena in the TLP measurement. The generated e-traps are permanent and can introduce detrimental conduction current harmful to the device performance. By properly selecting the buffer material, these defects can be removed.
\end{abstract}

Keywords: ESD; III-V; Reliability; Electron trapping; InGaAs; HEMT

\section{INTRODUCTION}

The requirement of high performance and low off-state power consumption in consumer electronics applications boosts the exploration of high mobility materials for future CMOS nodes [1]. III-V and Ge compounds are the tentative choice for $\mathrm{n}$ - and $\mathrm{p}$ - type channels, respectively [2]. Extremely high intrinsic electron mobility $\left(\sim 3000 \mathrm{~cm}^{2} / \mathrm{V}-\mathrm{s}\right)$ has been recently demonstrated by several groups $[3,4]$ in devices with InGaAs channel and $\mathrm{Al}_{2} \mathrm{O}_{3}$ gate oxide, with good interface quality (Dit $\sim 2 \times 10^{11} \mathrm{~cm}^{-2} \mathrm{eV}^{-1}$ [5]) and subthreshold swings ( $75 \mathrm{mV} /$ dec [3]). However, few studies on reliability have been reported [6] and ESD reliability has not been studied for III-V channel materials so far. In this paper, for the first time, a comprehensive study of planar $\mathrm{In}_{0.53} \mathrm{Ga}_{0.47} \mathrm{As}$ III-V devices reliability under ESD stress condition is conducted using both Transmission Line Pulse (TLP) and Pulse IV (P-IV) techniques. We found that the III$\mathrm{V}$ devices' TLP response is controlled by two types of defects from different physical origins. The generation mechanisms as well as the impact of device geometry and channel thickness are discussed in the paper. A unified model is proposed to explain the experimental observations. Finally, the impact of III-V buffer materials on ESD reliability is discussed.

The gate oxide of the device used in this work is $10 \mathrm{~nm}$ ALD $\mathrm{Al}_{2} \mathrm{O}_{3}$ with the TMA initial surface cleaning. The device received a $\left(\mathrm{NH}_{4}\right)_{2} \mathrm{~S}$ treatment prior to the gate oxide deposition. Forming gas annealing at $370{ }^{\circ} \mathrm{C}$ was performed on the finished devices. This ALD process reduces Ga and As oxidation on InGaAs surface and thus yield good quality interfaces. The detailed fabrication process flow and electrical characterization can be found in [3].

\section{TLP AND P-IV CHARCTERIZATION}

Conventionally, TLP technique is used to study the III-V device with InGaAs channel and InP buffer under ESD stress [7]. The typical result is shown in Fig. 1. Unlike the silicide blocked Si nMOS device which goes in snap-back at high current conduction [7], the III-V devices show a twostage current conduction during stress above Vt1. What is more, Ioff shows a 'walk-out' phenomenon. Conventional TLP technique can only monitor the off-state leakage current, Ioff. Besides, due to the limitation of the system, the Ioff measurement after ESD stress can only be performed after a fixed delay (e.g. 100ms). Hence, the information from TLP trace is not sufficient in understanding such phenomena we observed in III-V devices.

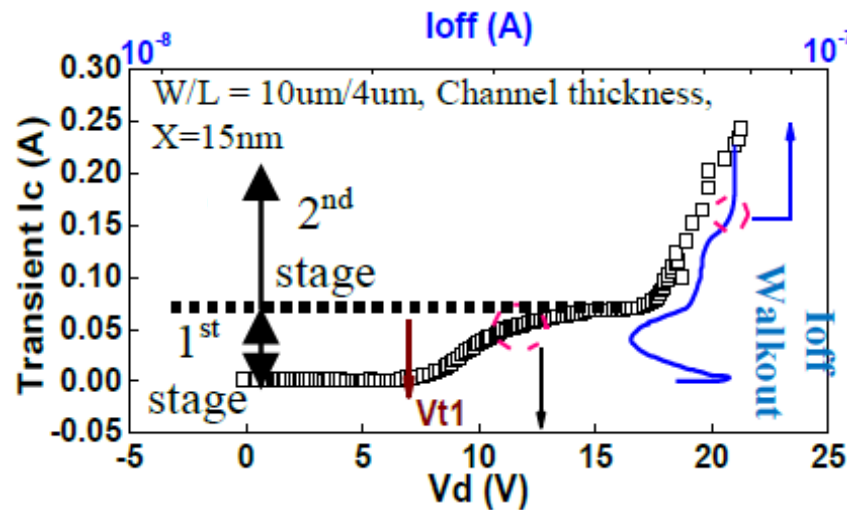

Fig. 1 Typical 200ns TLP response for an III-V InGaAs device with InP buffer layer. The device with channel thickness (X) of $15 \mathrm{~nm}$ is used.

A Pulsed IV technique (P-IV) is applied thereafter [8]. The connection of the P-IV system is shown in Fig. 2a. Three Keithley PMUs are connected to the device's three terminals to supply the required voltages or measure current in fast speed. The testing waveform is shown in Fig. 2 b. Similar to TLP technique, a 200ns stress pulse is applied on the drain. However, in P-IV, entire Idsat $\sim \mathrm{Vg}$ curves are measured after both short delay time $(20 \mu \mathrm{s})$ and long delay time $(5 \mathrm{~s})$, with $20 \mu \mathrm{s}$ measurement speed. By repeating this sequence with increasing stress $\mathrm{Vd}$, both the transient conduction current, Ic, and a series of Idsat $\sim \mathrm{Vg}$ curves can be obtained. In order to monitor the effect of defects created during stress close to the source and drain, both Forward Idsat $\sim \mathrm{Vg}$ (Forward IV: $\mathrm{Vd}=+2 \mathrm{~V}, \mathrm{Vs}_{\mathrm{s}}=0 \mathrm{~V}$ ) and Reverse Idsat $\sim \mathrm{Vg}$ (Reverse IV: $\mathrm{Vs}=+2 \mathrm{~V}, \mathrm{Vd}=0 \mathrm{~V})$ are measured. All the measurement sequences are controlled by Keithley KTEI software. 
Fig. 3 shows the typical results before and after stress. Before stress, the Forward and Reverse IV overlap each other, as shown in Fig.3a. However, Fig. 3b shows that when a high stress is applied, IVs measured after short delay time shift away from the fresh IV curves. When Vg is low, the Forward IV shifts to the negative direction while Reverse IV is hardly changed. However, when Vg becomes more positive, both IVs shift to the positive Vg direction. If measured after long delay time (5s), both IVs shifted in a positive $\mathrm{Vg}$ direction compared to the fresh condition, as shown in Fig.3c.

(a)

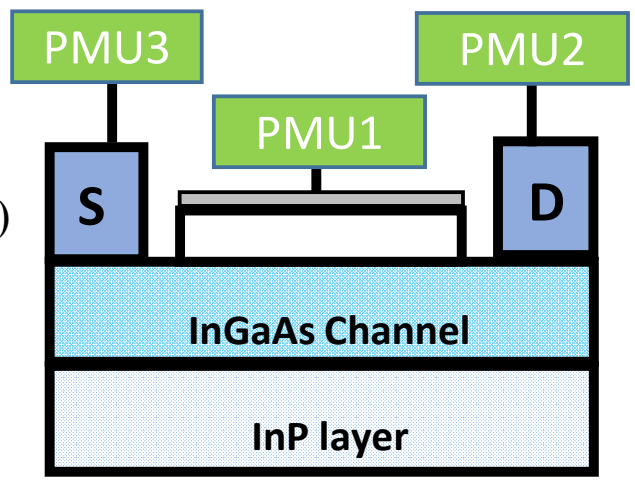

(b)

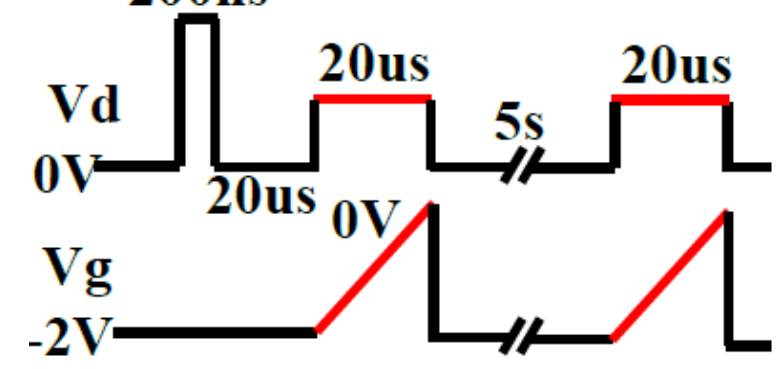

Fig. 2 (a) Pulsed Id-Vg (P-IV) measurement setup [8]: Keithley 4225 Pulse Measurement Units (PMUs) are used for both transient pulse stress and fast full Idsat-Vg measurement within $20 \mu \mathrm{s}$ or $5 \mathrm{~s}$ after stress. (b) P-IV waveform used in this work. Forward IV measurement is illustrated. Source is biased at $0 \mathrm{~V}$ all the time. For Reverse IV, the measurement pulse will be applied on the source side while Vd during measurement is kept as $0 \mathrm{~V}$.

\section{RESULT DISCUSSION}

\section{A. Defect creation during high current stress}

IVs can shift in both directions after stress as observed in Fig.3b\&c, indicating both positive and negative charge formation during the applied stress. Negative shifts can be observed in forward IVs with short delay time but disappear after long delay time, indicating that there exists positive charge formation and these positive charges are not stable.
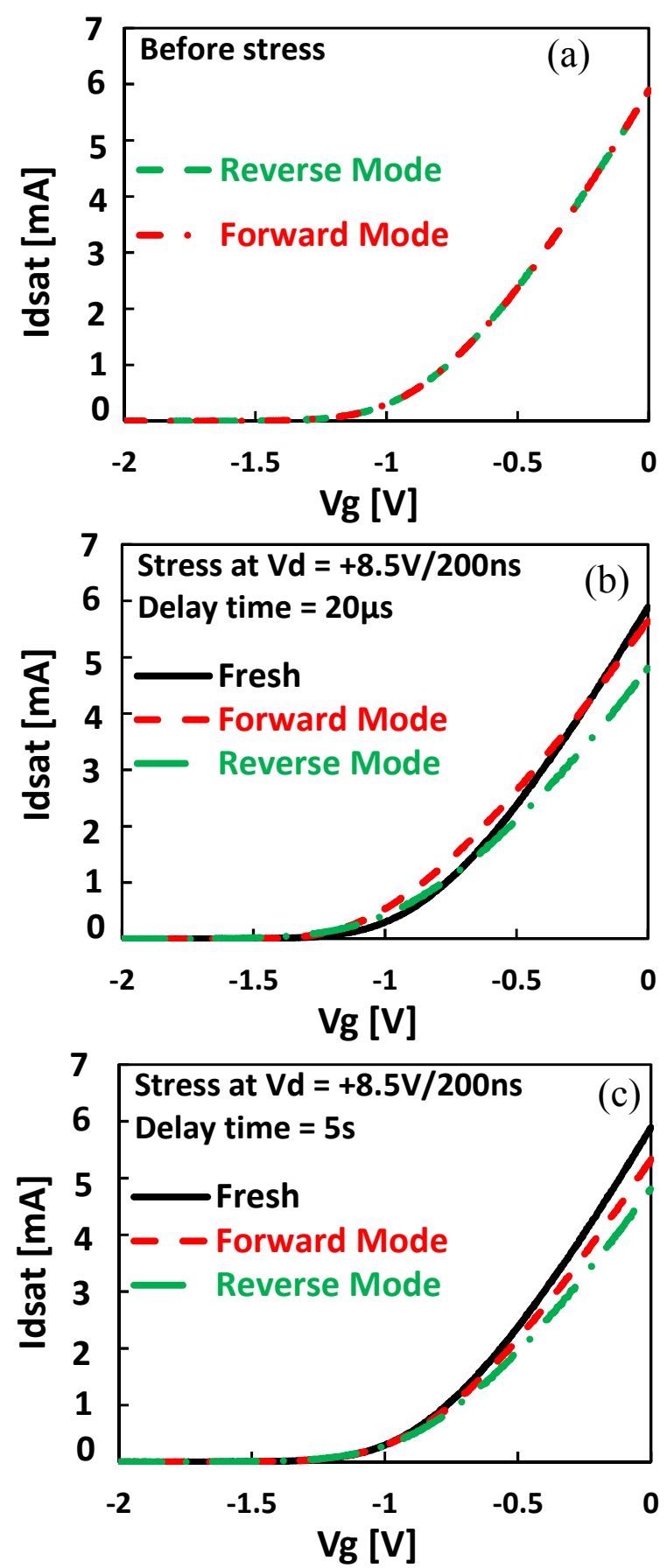

Fig. $3 I_{d s a t}-V_{g}$ curves measured before stress (a) and after high stress with $20 \mu \mathrm{s}$ (b) and $5 \mathrm{~s}$ (c) measurement delay. High transient stress $\mathrm{V}_{\mathrm{d}}$ of $+8.5 \mathrm{~V} / 200 \mathrm{~ns}$ is used. For measurements, $-2 \mathrm{~V}$ is applied at either the drain (Forward mode) or source side (Reverse mode). Measurement time is $20 \mu$ s.

D1 e-traps are detrapped during the TLP stress through transient Ef-lowering induced electron discharging as illustrated in Fig.5a. Before the pulsed stress, all D1 e-traps below Fermi Level (Ef) are filled. During the stress, Ef is temporarily lowered, leading to the electron de-trapping and thus manifesting as the positive charges w.r.t pre-stress condition. When the stress is removed, Ef will return to its 
stable level and the channel electrons gradually re-fill the de-trapped D1 e-traps, as confirmed in Fig.6.

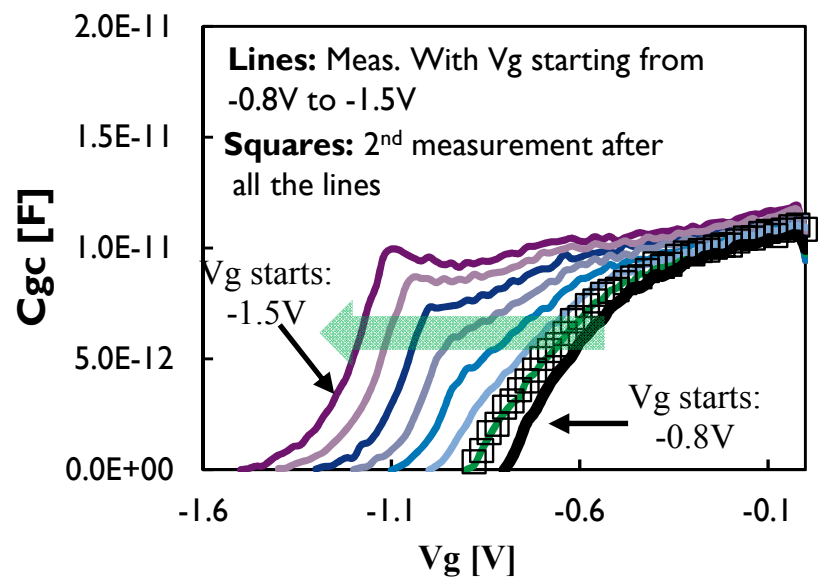

Fig. 4 Pulsed CV measured with Vg sweeping from different level to $0 \mathrm{~V}$ on one fresh device (the lines). After the measurement sweeping from $-1.5 \mathrm{~V}$, the device was then remeasured starting from $-0.8 \mathrm{~V}$ (' $\square$ '). The good agreement indicates no generation. The ramp rate of $150 \mathrm{kV} / \mathrm{s}$ is kept constant for all measurements.
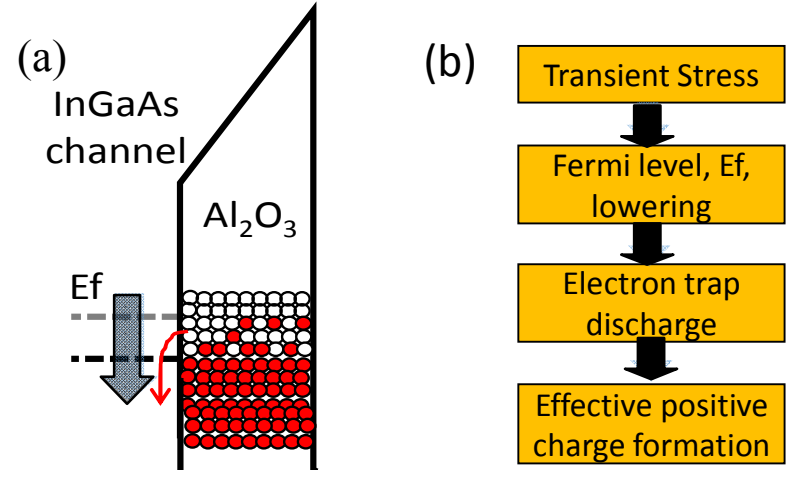

Fig. 5 (a) Energy band diagram during the stress. Fermi level lowering transiently can induce pre-existing e-traps discharging which manifest as the positive charge formation w.r.t pre-stress condition. (b) Flow chart of positive charge formation in the gate stack.

Fig.3c shows that positive shift can be observed in both Forward and Reverse IVs measured at long delay time after the stress, suggesting that negative charges have been formed during stress. Fig.3c also shows that the stressed IVs are not in parallel w.r.t the IV before stress. IVs after stress shift more positively at higher Vg. This indicates that these negative charges formed after stress can get discharged after long delay and then will gradually get charged when $\mathrm{Vg}$ increases. This also confirmed that these electron traps are generated by ESD stress rather than 'as-fabricated'. In the rest of the work, these electron traps are called the $\underline{\mathbf{D 2}} \mathbf{\mathrm { e }}-$ traps.

The generated D2 e-traps can be evaluated using the IV shift, $\Delta \mathrm{Vg}$, under high constant current as illustrated in the inset of Fig.7b. The impact of stress Vd pulse on D2 e-trap generation is shown in Fig. 7b and compared with the TLP graph (Ic $\sim \mathrm{Vd})$ in Fig. 7a. These generated D2 e-traps are closely related to the 1st-stage conduction during ESD stress, see TLP IV in Fig1.

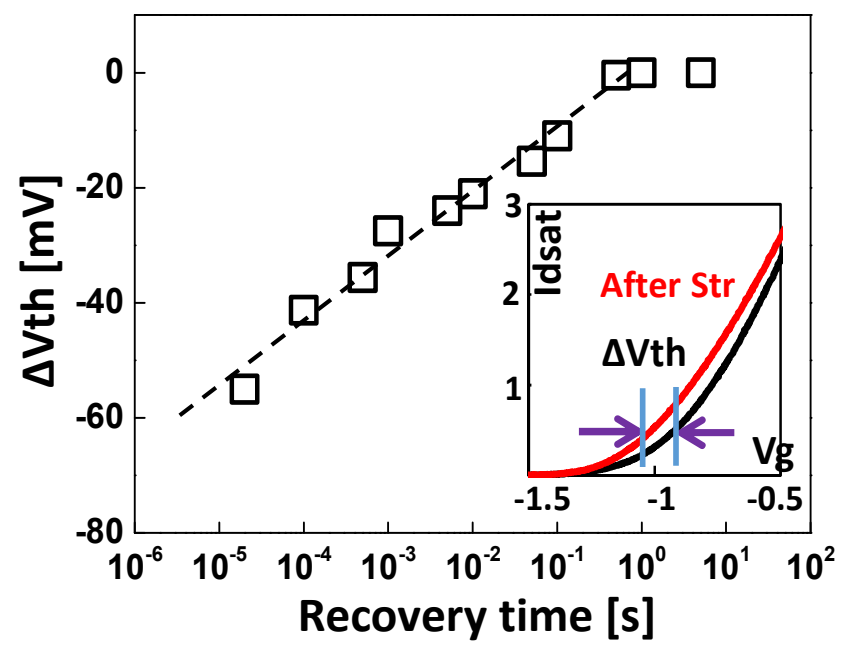

Fig. $6 \Delta \mathrm{V}$ th recovery under $\mathrm{V}_{\mathrm{g}}=-2 \mathrm{~V}, \mathrm{~V}_{\mathrm{d}}=0 \mathrm{~V}$ after a transient stress of $\mathrm{V}_{\mathrm{d}}=+8.5 \mathrm{~V}$ for $200 \mathrm{~ns}$. The shift is back to $0 \mathrm{~V}$ after $1 \mathrm{~s}$. Vth is extracted by constant current of $0.3 \mathrm{~mA}$ from Forward $\mathrm{I}_{\text {dsat }} \sim \mathrm{Vg}$ as shown in the inset.

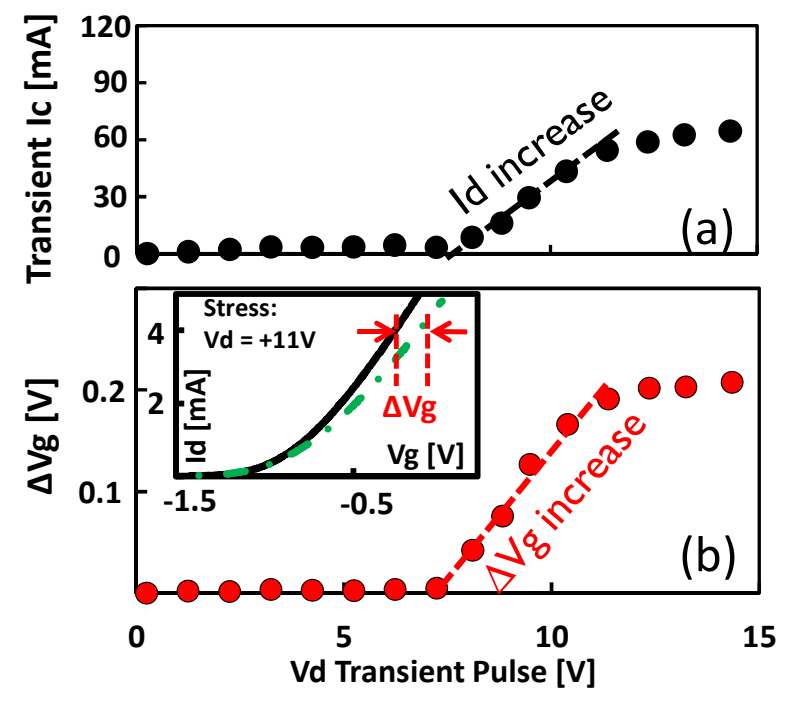

Fig. 7 Correlation between transient current $\mathrm{I}_{\mathrm{c}}$ (a) and $\Delta \mathrm{Vg}$ (b). $I_{c}$ is from the $1^{\text {st }}$ stage conduction in Fig. $1 . \Delta V g$ is extracted from long delay Reverse IV shifts under constant current of 4 $\mathrm{mA}$ as shown in the inset of (b).

Poole-Frenkel defects have been demonstrated to manifest in InP material at high field [9]. In order to understand the physical origin of these D2 e-traps, the TLP curves shown in Fig. 8a are re-plotted with $\log _{10}(\mathrm{Ic} / \mathrm{Vd}) \sim \mathrm{Vd}^{0.5}$. A linear relationship can be observed before saturation, as shown in Fig.8b. This indicates that the 1st-stage conduction is controlled by a Poole-Frenkel hopping mechanism through Frenkel defects (PF defect) [10]. During high stress, it is expected high electric field 
exists close to the drain. This is further confirmed by the TCAD simulation, as shown in Fig. 9. When a high voltage is applied on the drain side under device off-state, a high electric field can be observed (red region in Fig.9). The high electric field results in impact ionization and generates hot hole and electron pairs. The hot holes create interstitial deficiencies in the InP buffer near the drain which electrically behave as electron traps. The current reaches its saturation when all the defect sites are filled. The hot electrons will be attracted to flow into the drain since there is a large positive $\mathrm{Vd}$.

These PF defects are permanent and will not recover, as shown in Fig. 10. This means that as soon a PF current conduction is observed in the TLP IV plot, the devices has been damaged. This results in an extreme low $(\sim 0 \mathrm{~A})$ failure current (It2). The D2 e-traps can become neutral but the defect will be charged again at e.g. high Vg (see Fig.10)

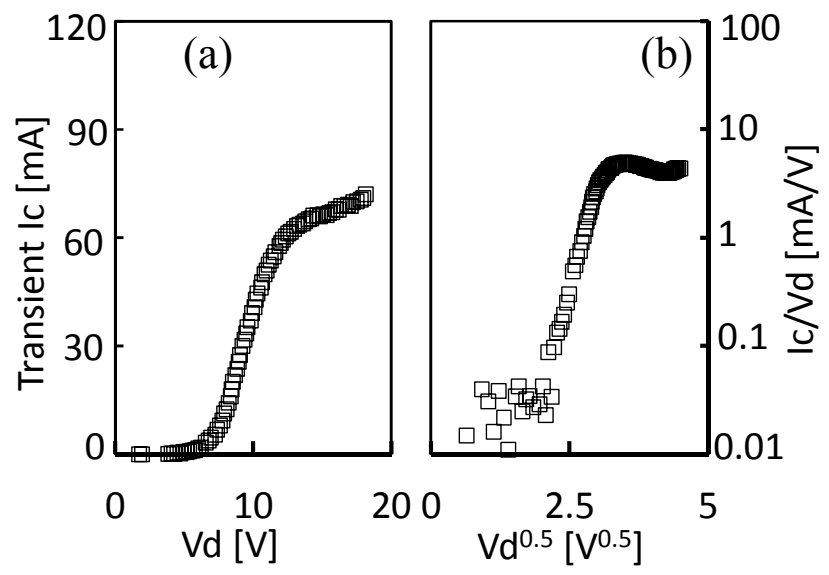

Fig. 8 (a) $1^{\text {st }}$ stage transient current Ic measured by TLP technique. (b) Linear relation between $\log _{10}(\mathrm{Ic} / \mathrm{Vd})$ and $\mathrm{Vd}^{0.5}$ is the signature of Frenkel defects (PF defects) generation [10].

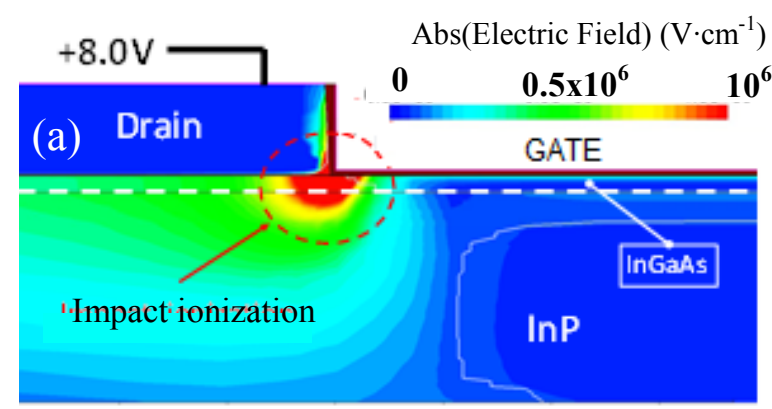

(b)

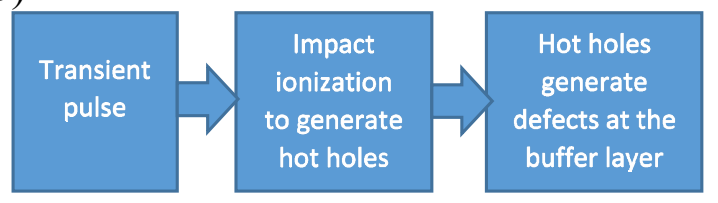

Fig. 9 (a) TCAD simulation and (b) flow chart for the explanation of negative charge formation in the buffer layer during transient stress: Impact ionization induced Frenkel defects (PF defects) formation.

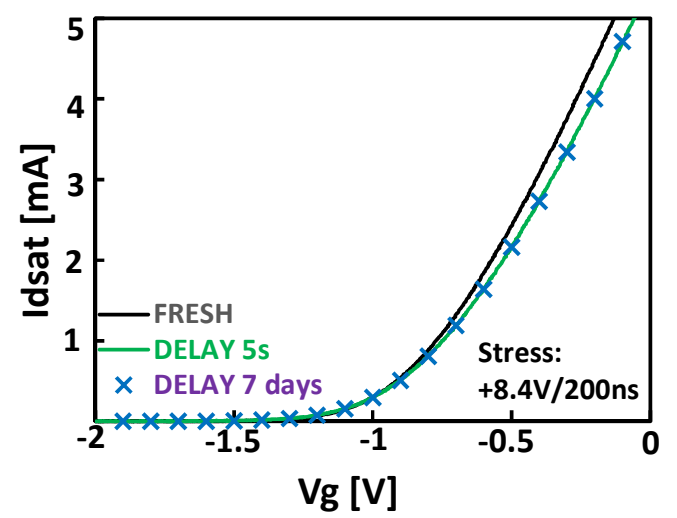

Fig. 10 Reversed IV curves before stress and after stress with $5 \mathrm{~s}$ and 7 days recovery. Non-parallel IV shift indicates the existence of electron charging and discharging between the channel and the InP buffer layer.

\section{B. Impact of channel length}

Fig.11a shows the TLP response from the devices with channel length $L$ varying from $1 \mu \mathrm{m}$ to $50 \mu \mathrm{m}$. To show the $\mathrm{L}$ dependence more clearly, the voltage, $\mathrm{Vd}$, extracted under the constant current of $20 \mathrm{~mA}$ is plotted against channel length as shown in Fig. 11b: the conduction starts early when the channel length reduces. This is also due to the enhanced electric field in shorter channel devices: Compared with long channel devices, the same electric field in shorter channel devices can be reached with lower Vd.
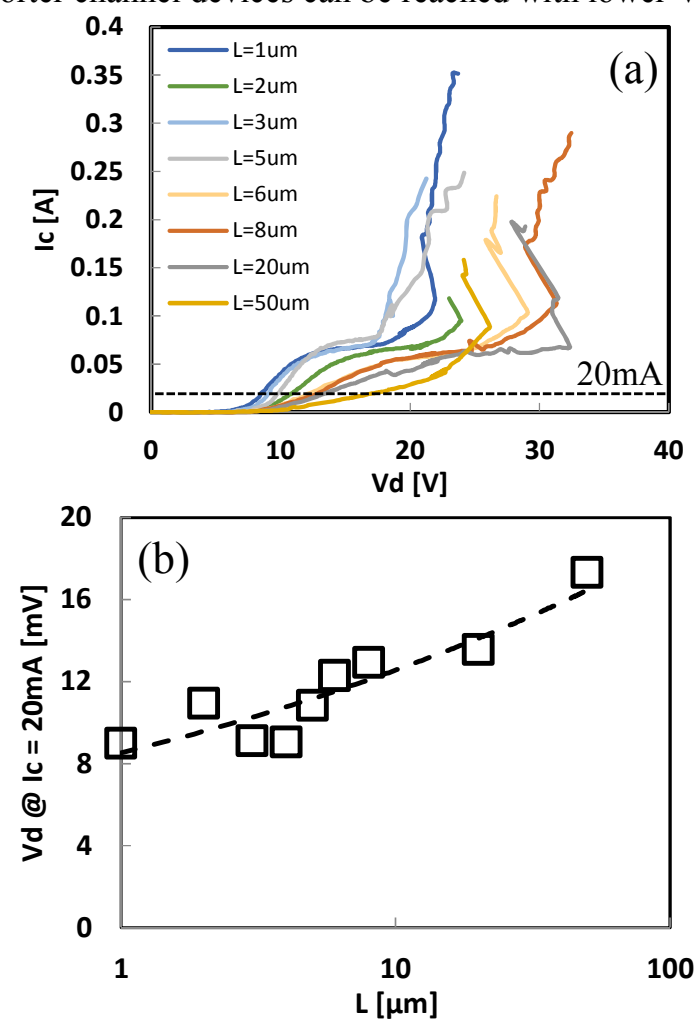

Fig. 11 (a) TLP response on devices with different channel length from 1 um to 50um. (b) Vd under the constant current of $20 \mathrm{~mA}$ taken from TLP responses in (a). 


\section{Channel thickness impact}

In this section, the impact of channel thickness $(\mathrm{X})$ on the TLP transient current, Ic, is checked. Fig. 12a shows that the $1^{\text {st }}$ stage conduction starts earlier when $\mathrm{X}$ reduces. The D2 e-traps generation is also found to start at same lower voltage as shown in Fig.12b, further supporting that D2 etraps are responsible for this conduction. The observed channel thickness dependence can be explained by the enhancement of the energy quantization in the thinner channel quantum well as confirmed by k.p simulations in Fig. 12d [3]. For thinner channels with stronger confinement, the electron energy becomes higher, leading to impact ionization under lower stress condition. Stronger confinement also lead to the large change of the Fermi level, leading to larger de-trapped D1 e-traps in thinner channel thickness as confirmed in Fig. 12c. Fig.12b shows that D2 e-traps saturated at the same level respective of the InGaAs channel thickness. This suggests that the total number of precursors for D2 e-traps is mainly determined by the InP buffer.
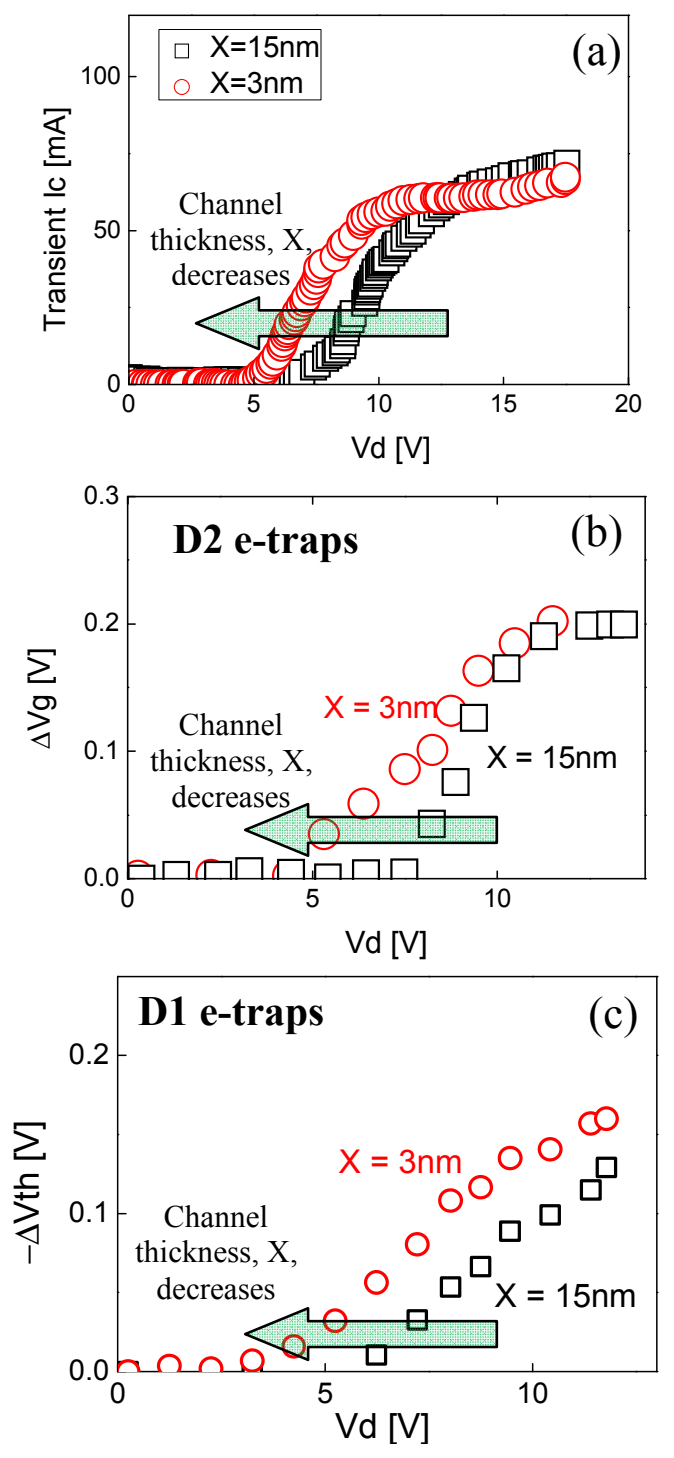

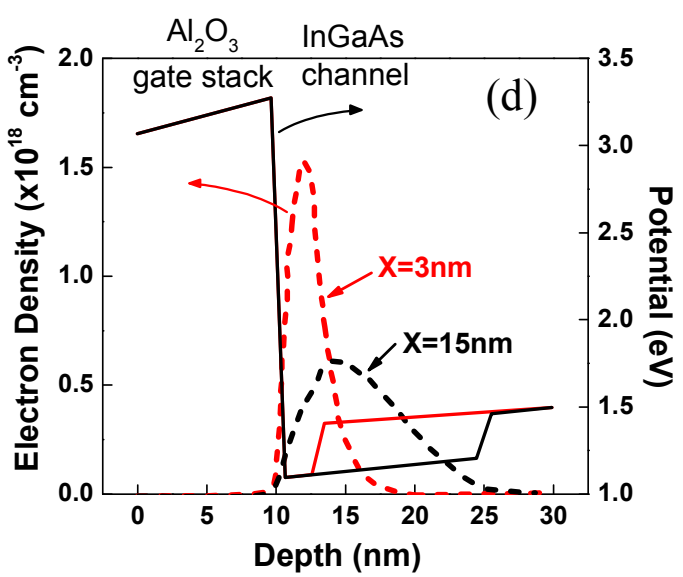

Fig. 12 (a) Conduction current during stress, Ic, for devices with different channel thickness (X). (b) The corresponding D2 e-traps generation for devices with different X. D2 e-traps are evaluated by $\Delta \mathrm{Vg}$ extracted in the same way as in Fig. $7 \mathrm{~b}$. (c) The corresponding D1 e-traps for devices with different X. $\mathrm{D} 1 \mathrm{e}$-traps are evaluated by $\Delta \mathrm{Vth}$ extracted in the same way as in Fig.6. (d) Energy quantization in the channel quantum well confirmed by k.p simulations [3]. For thinner channels, the electron energy becomes higher.

\section{Explanation of TLP IV curves after stress based on traps}

All the IV shifts after short and long delay time in Fig.3 can be well explained with the D1 and D2 e-traps, as illustrated in Fig. 13. After stress, with short delay (Fig.13a\&b), detrapped pre-existing D1 e-traps in the gate stack do not have enough time to be refilled and thus behave as positive charge (marked as "+") with respect to before-stress condition. The electrons captured by the generated D2 etraps in the InP buffer cannot escape in short time and thus exhibit as negative charge in the InP layer close to the drain (marked as "-"). IV measurements in Forward and Reverse mode pinch off the channel near the drain and source respectively leading to more negative IV shift in Forward mode. After long delay (Fig.13 c\&d), D1 e-traps are refilled. D2 e-traps are also full discharged and become neutral. However, they can get recharged by capturing channel electrons at higher $\mathrm{Vg}$ and thus lead to positive IV shift. Due to the different pinch-off region, Reverse IV exhibits more positive shift.

The experimental observation in the TLP response in Fig.1 can also be well explained with the D1 and D2 e-traps. Gate leakage current is negligible in our devices and therefore, the measured Ioff mainly comes from gate induced drain leakage current (GIDL). D1 e-traps are very close to the interface and thus can enhance the GIDL through remote trap-assisted tunneling [11]. The negatively charged D2 etraps in the buffer layer can behave like a back-gate, suppressing the local electric field near the drain side. Therefore, GIDL current will reduce. D1 e-traps will dominate when stress is low. However, D2 e-traps will gradually take over when stress increases. This can be further confirmed by the $\Delta \mathrm{V}$ th measured after different stress Vd, as shown in Fig.14. The opposite effect on Ioff 
from these two types of traps lead to the Ioff 'walk-out' measured in the TLP graph.

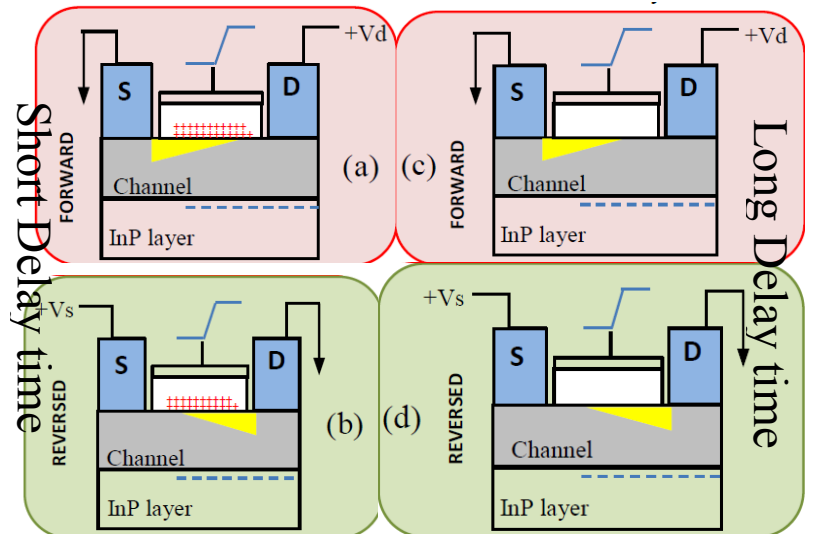

Fig. 13 Schematic explanation for IV curves shift shown in Fig.4 (b\&c). Detrapped pre-existing e-traps (D1) behaves as unstable positive charge in the gate stack, while the generated e-traps (D2) behave as negative charges in the buffer layer.

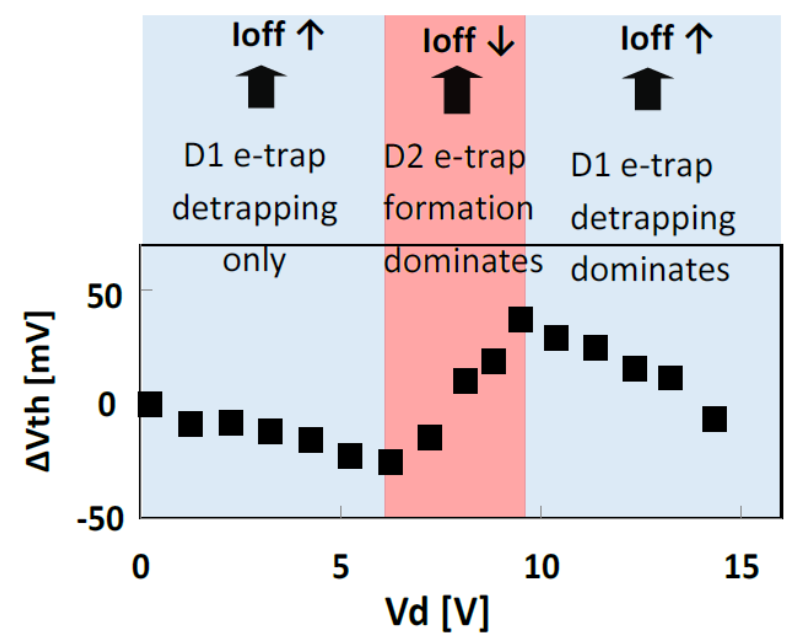

Fig. $14 \Delta V$ th measured after different stress $V d$. Vth is extracted from Reverse IV after 100ms measurement delay.

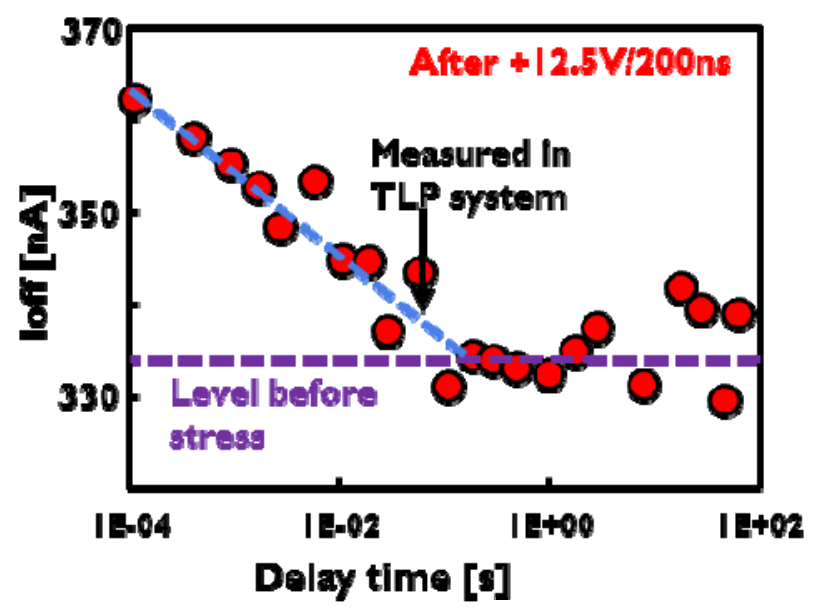

Fig. 15 Impact of delay time on the off state leakage current measurement. After $100 \mathrm{~ms}$ delay, Ioff dropped significantly compared with 100 us delay time.

It is expected that the re-filling of D1 e-traps (Fig. 6) will also lead to the reduction of Ioff current with longer delay time, as confirmed in Fig. 15. Therefore, Ioff current which is measured after $100 \mathrm{~ms}$ delay time in the typical TLP system has already been recovered. The wrong condition of measurement in TLP leads to the under-estimation of Ioff.

The $1^{\text {st }}$-stage conduction behavior during TLP measurement can also be explained by the Pool-Frankel conduction through D2 e-traps in the InP buffer layer which provides an effective current conduction path. There is no InP buffer in typical silicide blocked $\mathrm{Si}$ devices and therefore the $1^{\text {st }}$ conduction cannot be observed.

\section{E. High current conduction model}

Two-stage current conduction phenomenon observed in Fig.1 can be modelled in Fig. 14. The 1st-stage PoolFrankel conduction through the generated D2 e-traps can be considered as a resistor $\left(\mathrm{R}_{\mathrm{PF}}\right)$ across the source and drain in the InP buffer layer. The 2nd-stage can be modelled by a buffer resistor $\mathrm{R}_{\mathrm{B}}$ and parasitic bipolar transistor (BJT) formed between channel and buffer layer $(\mathrm{N}+$ type emitter, P-type buffer, $\mathrm{N}+$ type collector). $\mathrm{R}_{\mathrm{PF}}$ provides an effective current conduction path and as a consequence, voltage drop on $R_{B}$ is too small to trigger the BJT to turn on or "snapback" until the transient pulse increases significantly. Turn on of the BJT results in catastrophic failure.

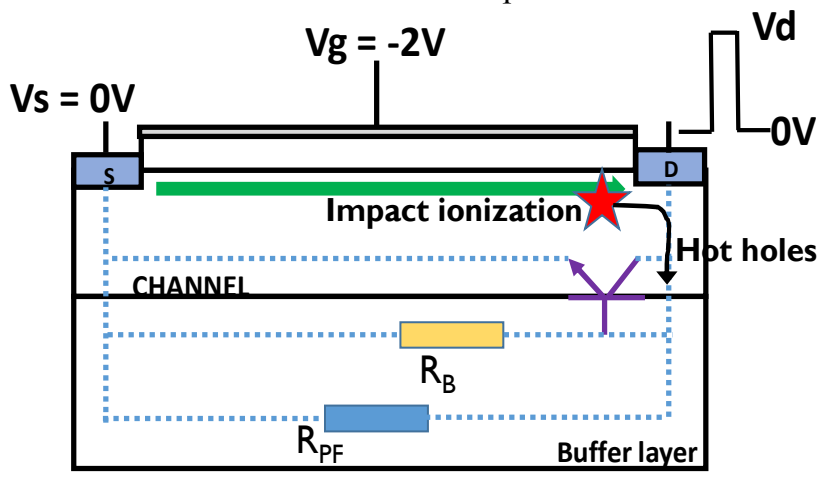

Fig. 15 General model to explain the conduction mechanism in III-V devices. Channel and buffer layer can form the parasitic NPN BJT which is in series connection with a buffer resistor, $\mathrm{R}_{\mathrm{B}}$. The PF defect induced conduction can be considered as a resistance, $R_{P F}$, between source and drain.

\section{F. Reliability improvement}

Since the detrimental D2 e-traps are in the InP layer, it is expected that these e-traps together with the $1^{\text {st }}$ stage conduction can be removed by replacing InP with a different buffer material. Fig. 16a shows a TLP response measured on one device with $\mathrm{p}$-InAlAs buffer. As expected, the $1^{\text {st }}$ conduction is gone and a snap-back is present. Also the walkout of the leakage current is gone as well, since there is no generation of D2 e-traps in the InAlAs buffer. The Forward and 
Reverse IVs are also shown in Fig.16b\&c. For short delay, the same negative shift still can be observed, suggesting the existence of the same pre-existing D1 e-traps. However, for long delay, the difference in IV is gone because there is no D2 e-traps generation.
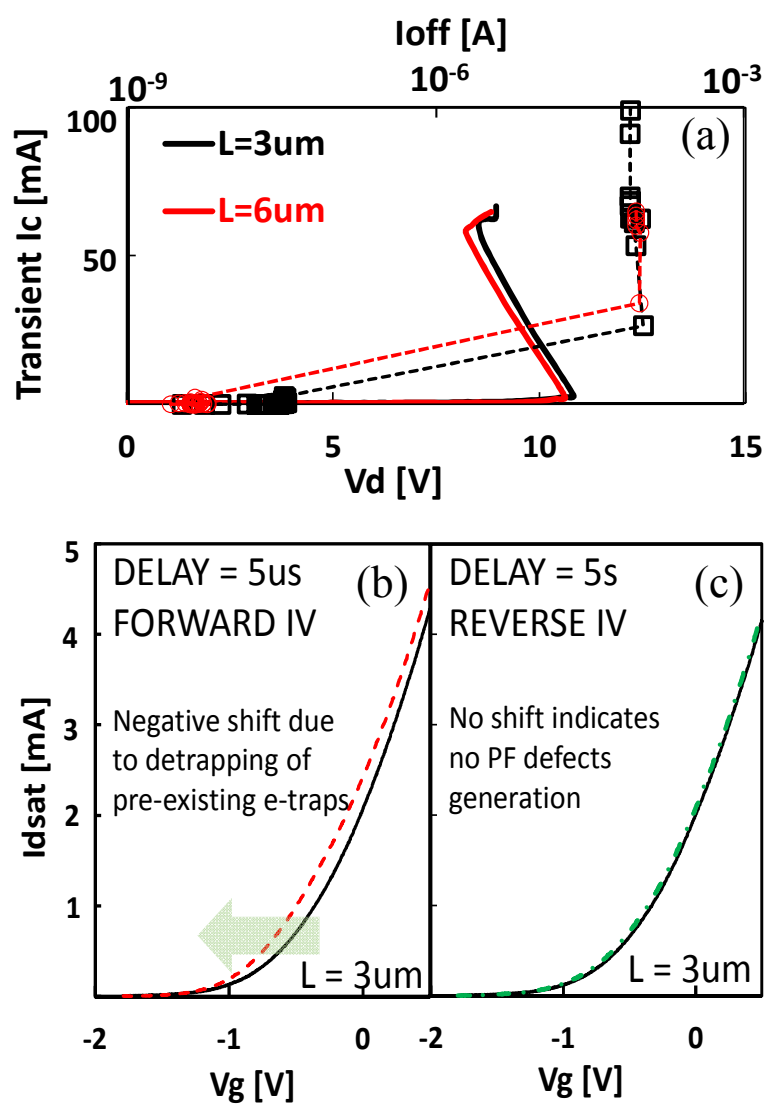

Fig. 15 Devices with p-InAlAs buffer layer. (a) Typical TLP graph under two different channel lengths. (b) The Forward IV measured after 5us delay. (c) The Reverse IV measured after $5 \mathrm{~s}$ delay.

\section{CONLUSION}

In the work, the TLP robustness of planar $\mathrm{In}_{0.53} \mathrm{Ga}_{0.47} \mathrm{As}$ devices with InP buffer layer is studied. Two-stage transient current conduction and off-state leakage current walk-out phenomena are found in the typical TLP response. Both phenomena are well explained by two types of traps formed through independent mechanisms: transient Ef-lowering induced pre-existing e-traps discharging in the gate stack (D1 e-traps) and hot hole induced Frenkel defects generation in the InP buffer through impact-ionization (D2 e-traps). The $1^{\text {st }}$ stage conduction is due to the generation of the D2 e-traps. We also found that the $1^{\text {st }}$ stage conduction will start at lower $\mathrm{Vd}$ voltage on devices with thinner channel thickness or shorter channel length, due to the enhancement of the electric field close to the drain. These Frankel defect (D2 e-traps) are permanent and can introduce a detrimental conduction current harmful to device performance. By properly selecting the buffer material, these permanent defects can be removed.
[1] G. Yeap, "Smart mobile SoCs driving the semiconductor industry: Technology trend, challenges and opportunities," in IEDM Tech. Dig., 2013, pp. 1.3.1-1.3.8.

[2] K. J. Kuhn, "Considerations for Ultimate CMOS Scaling," IEEE Trans. Electron Devices, vol. 59, pp. 18131828, 2012.

[3] A. Alian, M. A. Pourghaderi, Y. Mols, M. Cantoro, T. Ivanov, N. Collaert, et al., "Impact of the channel thickness on the performance of ultrathin InGaAs channel MOSFET devices," in IEDM Tech. Dig., 2013, pp. 16.6.1-16.6.4.

[4] S. Takagi and M. Takenaka, "High mobility CMOS technologies using III-V/Ge channels on Si platform," in Ultimate Integration on Silicon (ULIS), 2012, pp. 1-4.

[5] T. Hoshii, S. Lee, R. Suzuki, N. Taoka, M. Yokoyama, H. Yamada, et al., "Reduction in interface state density of A12O3/InGaAs metal-oxide-semiconductor interfaces by InGaAs surface nitridation," J. Appl. Phys., vol. 112, pp. -, 2012.

[6] J. Franco, A. Alian, B. Kaczer, D. Lin, T. Ivanov, A. Pourghaderi, et al., "Suitability of high-k gate oxides for IIIV devices: A PBTI study in In0.53Ga0.47As devices with A12O3," in Proc. IRPS, 2014, pp. 6A.2.1-6A.2.6.

[7] T. J. Maloney and N. Khurana, "Transmission line pulsing techniques for circuit modelling of ESD phenomena," in EOS/ESD, 1985, pp. 49-54.

[8] Z. Ji, S. W. M. Hatta, J. F. Zhang, W. Zhang, J. Niblock, P. Bachmayr, et al., "A new technique for probing the energy distribution of positive charges in gate dielectric," in Microelectronic Test Structures (ICMTS), 2014 International Conference on, 2014, pp. 73-78.

[9] A. Polity and T. Engelbrecht, "Defects in electronirradiated InP studied by positron lifetime spectroscopy," Physical Review B, vol. 55, pp. 10480-10486, 04/15/ 1997.

[10] J. S. Brockman, L. Gao, B. Hughes, C. T. Rettner, M. G. Samant, K. P. Roche, et al., "Subnanosecond incubation times for electric-field-induced metallization of a correlated electron oxide," Nat Nano, vol. 9, pp. 453-458, 06//print 2014.

[11] M. Gurfinkel, J. S. Suehle, J. B. Bernstein, and Y. Shapira, "Enhanced Gate Induced Drain Leakage Current in HfO2 MOSFETs due to Remote Interface Trap-Assisted Tunneling," in IEDM Tech. Dig., 2006, pp. 1-4. 